\title{
The Effect of the Immunosuppressive Drug Sirolimus on structure of the Submandibular Salivary Glands of the Rat
}

\author{
Salah A. Soliman', Laila S. Ghali and Rasha M. Taha \\ ${ }^{1}$ Oral Biology Dept., Faculty of Oral and Dental Medicine Sinai university, Egypt. ${ }^{2}$ Oral Biology Dept., Faculty of \\ Oral and Dental Medicine Cairo university, Egypt. ${ }^{3}$ Oral Biology Dept., Faculty of dentistry, Suez Canal university, \\ Egypt
}

\begin{abstract}
Background: Submandibular salivary glands are responsible for secretion of major amount of saliva which is important for normal oral environment of the oral cavity. Aim: to evaluate the effect of the Immunosuppressive drug Sirolimus on structure and immunohistochemistry of the submandibular salivary glands of the rats. Material and Methods Forty healthy adult male albino rats (body weight 150 -180 gram) were divided into two equal groups 20 animals each. Group 1 served as controls while group 2 were treated with the immunosuppressive drug sirolimus (rapamycin). Control group were subdivided into two subgroups subgroup 1.1: received ethanol and saline in comparable volume to group 2 and same route of administration while subgroup 1.2 were left untreated. After 3 months, rats were sacrificed and specimens of the right side of submandibular salivary glands were stained with $\mathrm{H} \& \mathrm{E}$ and Immunoperoxidase. While specimens of the left side were examined with transmission electron microscope. Results: Histologically, by H \& E, both control subgroups showed normal structure of submandibular salivary gland. While, rats administrated sirolimus showed altered structure. Using TEM, the ultrastructural of submandibular salivary glands of both control subgroups showed normal architecture. While, sirolimus treatedgroup revealed degeneration in the glands acinar and ductal cells. Immunohistochemical findings showed normal staining reactivity of submandibular salivary gland of the control group while, sirolimus-treated group showed marked reduction in their staining reaction. Conclusion: Sirolimus administration cause structural and ultrastructural alterations in the parenchymal and stromal elements of the submandibular salivary gland that affect the salivary secretion and may lead to detrimental effect on oral health.
\end{abstract}

Key words: Immunosuppressives, sirolimus, salivary glands

\section{Introduction}

Saliva is biological fluid, that gained a lot of interest because of its physiologic diagnostic medium. Its major components include a broad spectrum of proteins and peptides, nucleic acids, electrolytes, and hormones $^{(1)}$. Saliva can encourage soft tissue repair by reducing clotting time and accelerating wound contraction. A major protective function results from the salivary role in maintenance of the ecological balance in the oral cavity ${ }^{(2)}$. Loss of saliva results in a variety of difficulties and miseries. saliva is important in preparing food for mastication, for swallowing, and for 
normal taste perception. Without saliva, mealtimes are difficult, uncomfortable, and embarrassing. The complex mix of salivary constituents provides an effective set of systems for lubricating and protecting the soft and hard tissues. Protection of soft tissues is afforded against desiccation, penetration, ulceration, and potential carcinogens by mucin and anti-proteases(3). The submandibular salivary gland is anatomically, most complex of the major salivary gland and responsible for secretion of great protein of saliva so it was be chosen to be the tissue of study. Immunomodulators act at different levels of the immune system. Therefore, different kinds of drugs have been developed that selectively either inhibit or intensify the specific populations and subpopulations of immune responsive cells, i.e. lymphocytes, macrophages, neutrophils, natural killer (NK) cells, and cytotoxic T lymphocytes (CTL). Immunomodulators affect the cells producing soluble mediators such as cytokines $^{(4)}$. Sirolimus is widely used immunosuppressive drug for prevention of graft rejection in organ and tissue transplantation and treatment of cancer.

\section{Materials and Methods}

Forty healthy adult male albino rats with body weight ranging from 150 -180 gram were used in this investigation. The animals were divided into two equal groups 20 animals each. as follows. Group 1: animals served as controls and were subdivided to two subgroups, 10 animals each. subgroup 1.1: animals received ethanol and saline in comparable volume to group 2 and same route of administration. subgroup 1.2: animals were left untreated. Group2: animals were treated with the immunosuppressive drug sirolimus (rapamycin) dissolved in ethanol and saline in daily oral dose of $10 \mathrm{mg} / \mathrm{kg}$ body weight using a curved metallic oropharyngeal tube ${ }^{(5)}$. Sirolimus was supplied in the form of tablets that were ground into powder and dissolved in ethanol to a concentration of 1 $\mathrm{mg} / \mathrm{ml}$ and then diluted with saline to a final concentration of $0.5 \mathrm{mg} / \mathrm{ml}$. The animals were supplied standard natural diet and drinking tap water ad libium for the whole experiment period which lasted for three months. The percentage of mortality for each group was calculated and recorded. Gross examination of head and neck of each animal was carried out through the whole experimental period. At the end of experiment the rats of different groups were sacrificed by cervical dislocation. the submandibular salivary glands were dissected out and separated. Specimens of the right side were fixed in $10 \%$ neutral buffered formalin, processed, embedded in paraffin. Sections of 5-6 microns thick were cut and stained with: 1Hematoxylin and eosin to detect any possible structural changes in parenchymal and stromal elements of the glands. 2-Immunoperoxidase staining procedure for immunohistochemical localization of cytokeratin 5,6,18 using monoclonal mouse antibody. While specimens of the left side were used to be examined with transmission electron microscope. Negative controls: For negative control sections, primary antibody was substituted by nonspecific serum of the same dilution as its respective primary antibody and the procedures were continued as usual. Histological and immunohistochemical results were examined under the light microscope. The intensity of immunohistochemical staining results was assessed semi quantitively and scored as follows: ve for negative staining reactions, (1+) for weakly positive staining reactions. $2(2++)$ 
for moderately positive staining reactions. $\left(3^{+++}\right)$strongly staining reactions.

Preparation of specimens for transmission electron microscope examination:

Small sized $\left(1^{3} \mathrm{~mm}\right)$ samples were taken from the submandibular salivary glands of the left side of the animals of different groups and prepared for transmission electron microscope examination as follows: Specimens were rapidly fixed in 3\% phosphate buffered glutaraldehyde $(\mathrm{pH}$ 7.2) for 1-2 hours (primary fixation). Specimens were washed in 3 changes of phosphate buffer ( $\mathrm{PH} 7.2) 15$ minutes each. Specimens were post fixed in $1 \%$ buffered osmium tetroxide at $4^{\circ} \mathrm{C}$ for $1-2$ hours and then washed thoroughly in the buffer. Specimens were then dehydrated in ascending grades of ethanol. Final dehydration and clearing were carried out in propylene oxide for $1 / 2$ hour. Embedding was then carried out in oven-dried gelatin capsules using fresh epoxy resin and left to polymerize in an oven at $60^{\circ} \mathrm{C}$ for $24-36$ hours. semithin sections (1-2 microns thick) were cut stained with toluidine blue to be examined by the electron microscope. Ultrathin sections ( 0.06 microns) were cut using ultramicrotome and glass knives and then mounted on copper grids. Staining was carried out with saturated uranyl acetate in 50\% ethanol for 30 minutes, and then washed several times in distilled water followed by staining with

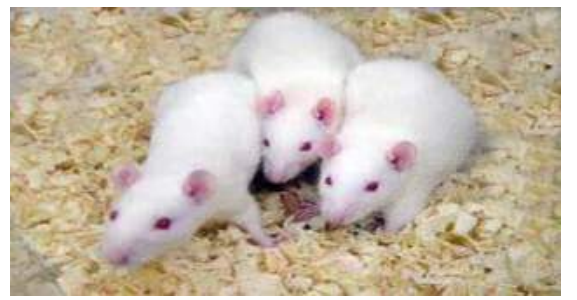

Fig. 1: rats of the control group showing normal skin and hair distribution. lead citrate for 5 minutes. Finally, examination was carried out using the TEM (JEOL1000) in faculty of agriculture, Cairo University.

\section{Results}

I-percentage of mortality:

percentage of mortality of rats or both control and treated groups was $0 \%$.

\section{Il-Clinical finding:}

A-control group: animals of both subgroup 1.1\&1.2 which served as a control showed normal skin and hair distribution (Fig 1).

B- Sirolimus treated group showed huge areas of alopecia on the back \& thigh regions. (Fig 2).

\section{III-Light microscope results:}

1- Hematoxylin \& eosin stained sections: A-Control groups: Both control groups 1.1 and 1.2 showed similar normal structure of all salivary gland elements (Figures 3-5).

B-Sirolimus treated group (group II): Animals treated with the immunosuppressive drug sirolimus showed marked degenerative changes in their submandibular salivary gland elements (Figures 610).

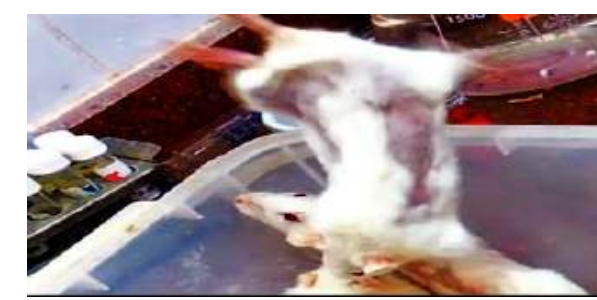

Fig. 2: sirolimus treated rat showing huge areas of alopecia on the back \& thigh regions. 


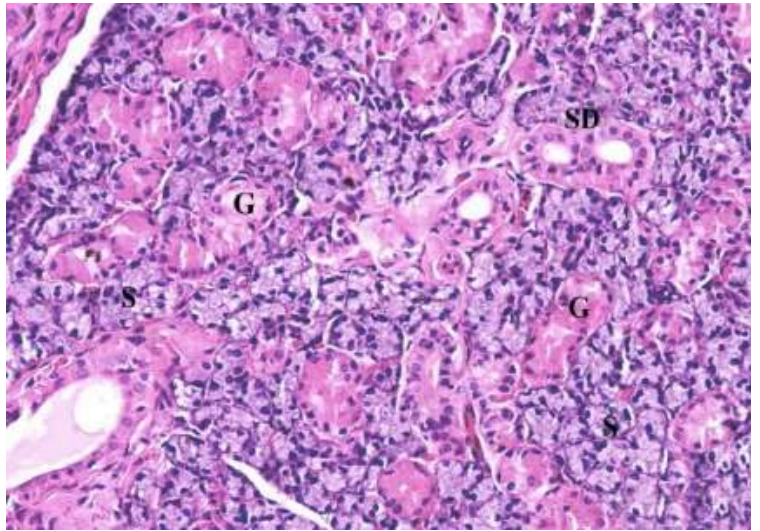

Fig. 3: submandibular salivary gland of a rat of the control group (1.1) showing normal serous terminal portions (S), striated ducts (SD) and granular convoluted tubules (G) . (H\&E.orig.mag.200)

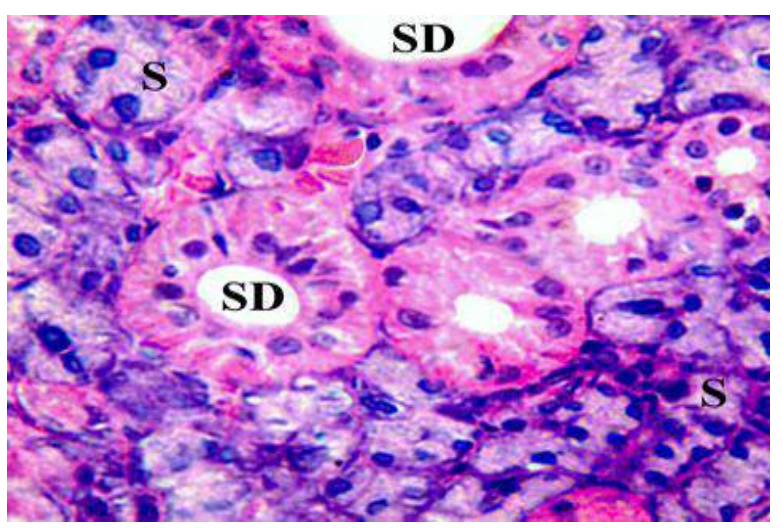

Fig. 4: submandibular salivary gland of a rat of the control group (1.1) showing serous terminal portions (S) and striated duct (SD) with its basal striation. (H\&E.orig.mag.400)

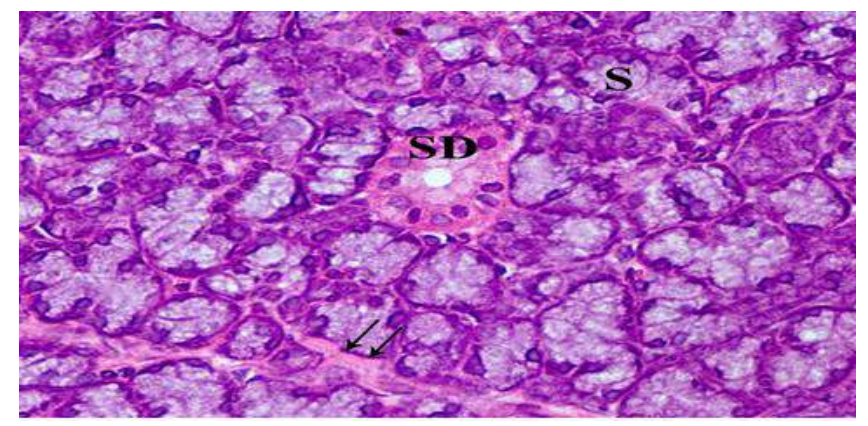

Fig. 5: submandibular salivary gland of a rat of the control group (1.1) showing normal serous terminal portions (S), C.T septa between lobules (arrow) and striated ducts (SD). (H\&E.orig.mag.250)

2- Immunohistochemical finding: Immunohistochemical staining reaction of submandibular salivary gland incubated with anticytokeratin number 5, 6 and 18 antibodies

Group I (control groups): Examination of sections taken from the control rats (subgroup $1.1 \quad \& 1.2$ ) revealed similar staining reactivity as follows (Fig 11). Group II (sirolimus treated group): Examination of sections taken from submandibular salivary gland of sirolimus treated group incubated with anticytokeratin monoclonal antibody cytokeratin 5,6,18 showed decreased in staining reaction of salivary gland elements (Fig 12).
IV-Electron microscope results:

Control groups: The submandibular salivary gland of both control groups (1.1) and (1.2) revealed normal ultrastructural findings (Fig. 13-18).

Group II (Sirolimus treated group): Electron microscopic examination of submandibular salivary glands of sirolimus treated rats revealed marked degenerative changes in ultrastructure of its parenchymal and stromal elements (Fig. 19-24).

\section{V-Cytokeratin staining reactivity} the cytokeratin staining reactivity of parenchymal and stromal elements of submandibular salivary gland of different groups is demonstrated in table (1). 


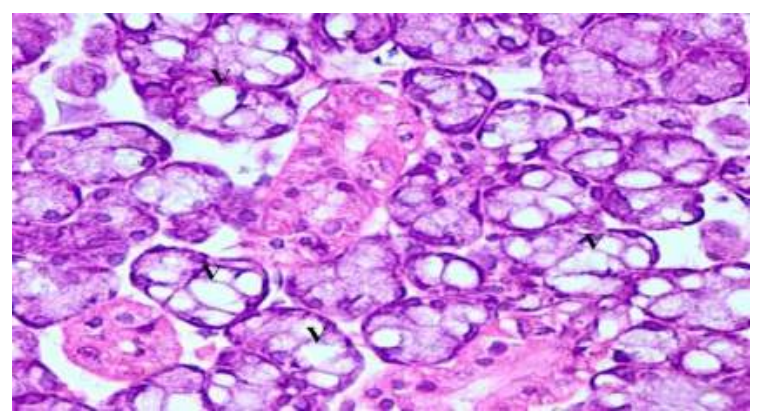

Fig.6: submandibular salivary gland of sirolimus treated group showing cytoplasmic vacuolization (V) of serous acinar cells and granular convoluted tubules (H\&E.orig.mag.400)

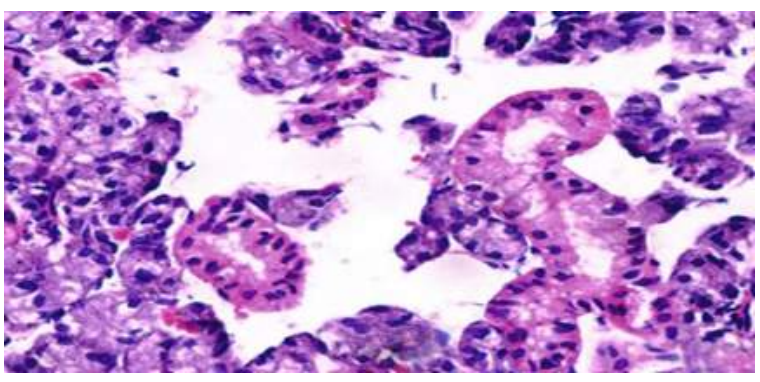

Fig.7: submandibular salivary gland sirolimus treated rat showing complete degeneration of wide areas of the parenchymal \& and stromal elements

(H\&E.orig.mag.250).

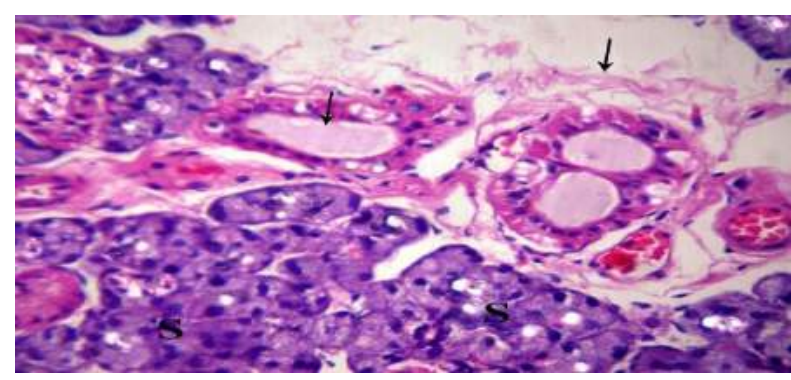

Fig. 9: submandibular salivary gland of sirolimus treated rat showing cytoplasmic vacuolization of serous acinar cells (S), and ducts, with stagnation of secretion in exe cratory duct and dissociation of $\mathrm{C}$, T septa (arrows). (H\&E.orig.mag.250)

\section{Discussion}

The present investigation studied the effect of sirolimus on submandibular salivary gland of albino rates. The submandibular salivary gland were selected as the target of the present research work because their main function is production of remarkable volume of saliva and the role of saliva on oral health is well appreciated. The submandibular salivary gland was chosen in

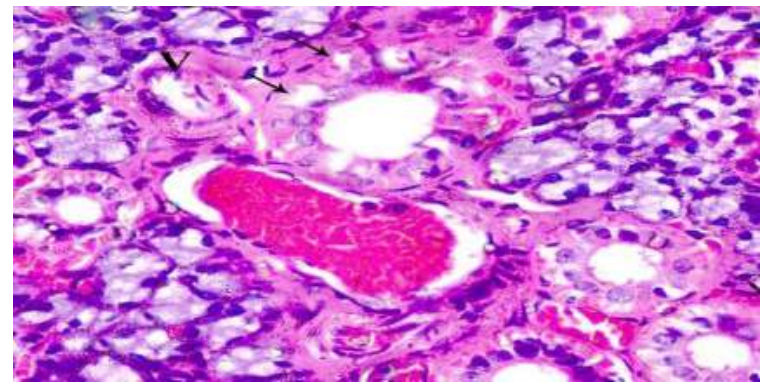

Fig. 8: submandibular salivary gland of sirolimus treated rat showing dilated blood vessels \& cytoplasmic vacuolization of ductal \& acinar cells $(V)$ with subtotal loss of basal striation of striated ducts (arrows). (H\&E.orig.mag.250)

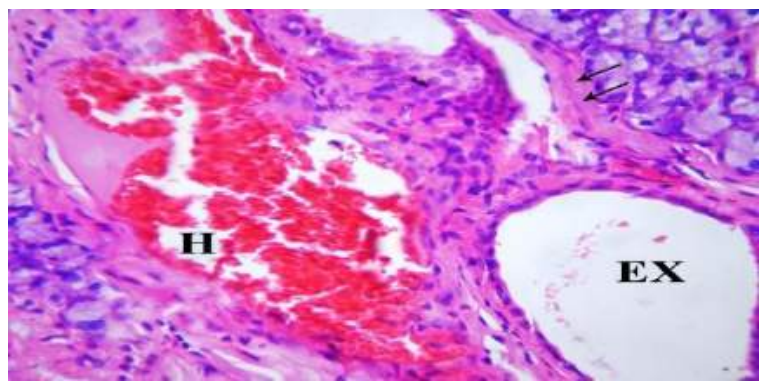

Fig. 10: submandibular salivary gland of sirolimus treated rat showing areas of hemorrhage $(H)$, halynization of connective tissue (arrows) notice the marked reduction in the height of the cells lining the execratory duct, the cells are almost flattened (EX).

(H\&E.orig.mag.400)

this investigation, because it is anatomically, most complex of the major salivary gland and responsible for secretion of great protein of saliva. Rats were chosen because they were easy to handle and manipulate and very resistant to infection that accompany their immunosuppression condition. Male rats were chosen to exclude any hormonal changes of female rats that my affect the results. In addition, the GCT cells exhibit greater volume in males than 
in females. Also, they possess a higher total volume of the endoplasmic reticulum, Golgi complex and secretory granules. The secretory granules are more developed in

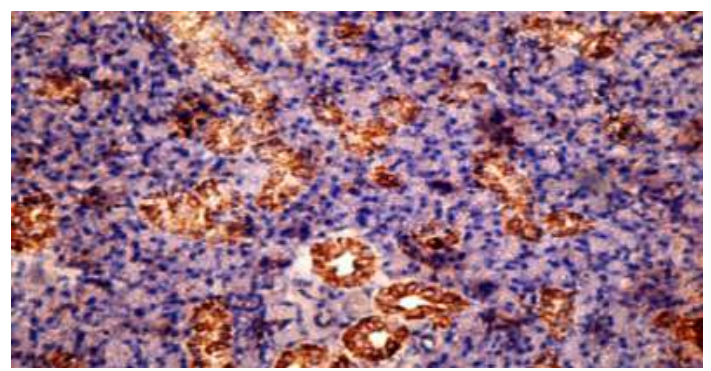

Fig. 11: submandibular salivary gland of rat of control group, and incubated with anticytokeratin $A b$, showing moderately positive staining reactivity in the serous acinar cells and granular convoluted tubules, strongly positive reaction of ductal cells. (origin mag 200). the male cells. So, any changes in the granular content of the GCTs could be easily detected in male rats more than in female (6).

Table 1: Cytokeratin staining reactivity

\begin{tabular}{|c|c|c|c|c|c|c|c|c|}
\hline $\begin{array}{l}\frac{0}{3} \\
\frac{0}{v}\end{array}$ & 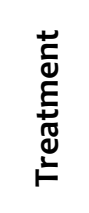 & 高 & 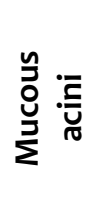 & 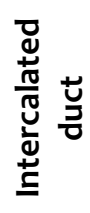 & 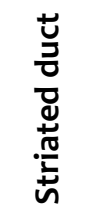 & 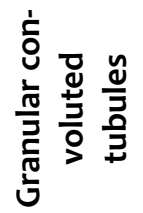 & 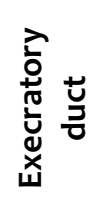 & 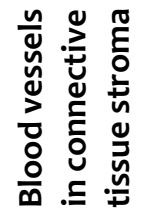 \\
\hline 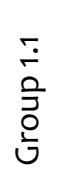 & 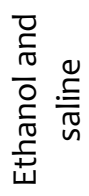 & $2+$ & -ve & $2+/ 3+$ & $2+/ 3+$ & $2+/ 3+$ & $2+/ 3+$ & $3+$ \\
\hline 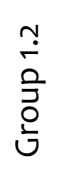 & 올 & $2+$ & -ve & $2+/ 3+$ & $2+/ 3+$ & $2+/ 3+$ & $2+/ 3+$ & $3+$ \\
\hline $\begin{array}{l}\text { N } \\
\frac{0}{7} \\
\text { 는 }\end{array}$ & 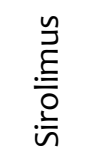 & $-1+$ & -ve & $1+/ 2+$ & $1+/ 2+$ & $1+/ 2+$ & $1+/ 2+$ & $2+$ \\
\hline
\end{tabular}

NB: Treatment duration $=3$ months

In the present investigation we used sirolimus as immunosuppression drug of choice because sirolimus has an advantage over calcineurin inhibitors that it has low toxicity toward kidneys. Transplant patients maintained on long-term calcineurin inhibitors tend to

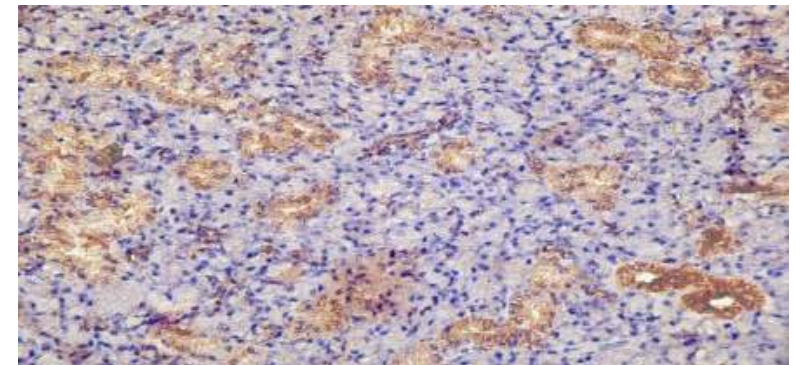

Fig. 12: A photomicrograph of submandibular salivary gland of sirolimus treated rats, showing negative reaction in the serous acinar cells and weakly to moderately positive reaction of the ductal cells and GCT . (origin mag 200) 
sirolimus treated group was purely sirolimus induced. The histological results of the present study revealed that the administration of sirolimus as immunosuppressive drug resulted in severe atrophic and degenerative changes in submandibular salivary gland most probably due to disturbance in the

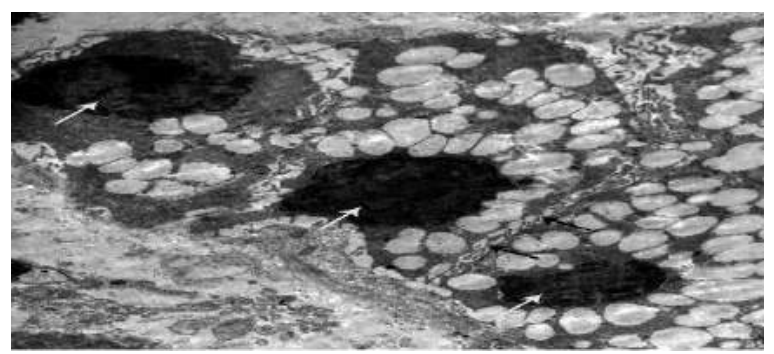

Fig. 13: An electron photomicrograph of the submandibular salivary gland of rat of control group (1.1) showing pyramidal serous cells with nucleus (white arrows) and secretory granules, cellular junction between acinar cells (black arrows). (uranyl acetale \& lead citrate×5000).

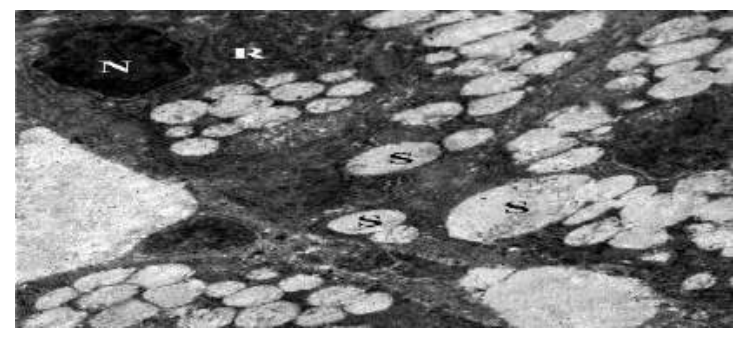

Fig.15: An electron photomicrograph of the submandibular salivary gland of rat of control group (1.1)

showing serous cells with nucleus $(N)$ and rough endoplasmic reticulum $(R)$ and secretory granules (S).(uranyl acetale \& lead citrate $\times 8000$ )

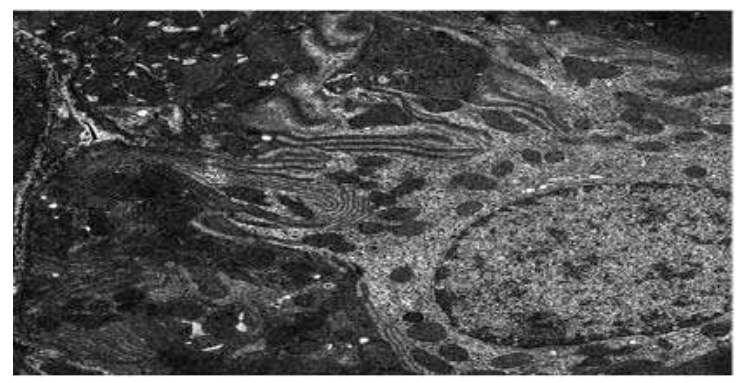

Fig.17: An electron photomicrograph of the submandibular salivary gland of rat of control group (1.2) showing striated duct with basal infoldings and a lot of mitochondria. (uranyl acetale \& lead citrate×8000) metabolic activity that results in adverse changes in the structure of acinar cells, duct system and associated investing stroma. The atrophy of the secretory portion was demonstrated through the intracytoplasmic vacuolization which was confirmed also in the ultrastructural findings.

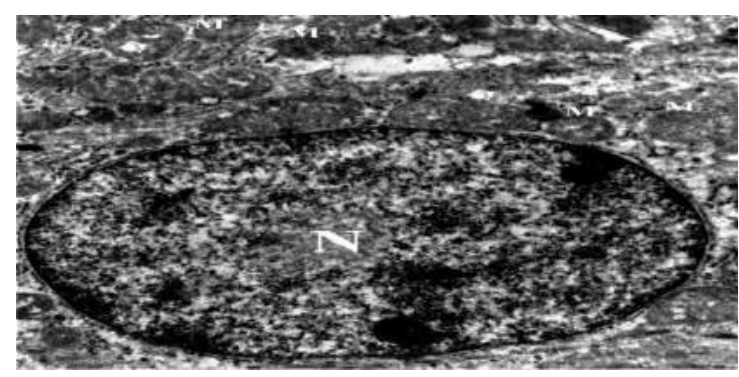

Fig. 14: An electron photomicrograph of the submandibular salivary gland of rat of control group (1.2) showing cell of intercalated duct with large euchromatic nucleus ( $N$ ) and cytoplasm with a lot of mitochondria (M). (uranyl acetale \& lead citrate×12000).

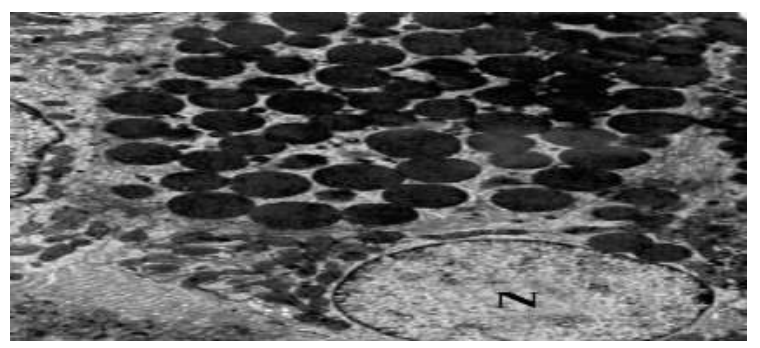

Fig. 16: An electron photomicrograph of the submandibular salivary gland of rat of control group (1.2) showing granular convoluted tubules with nucleus $(N)$ and a lot of electron dense granules. (uranyl acetale \& lead citrate $\times 8000)$

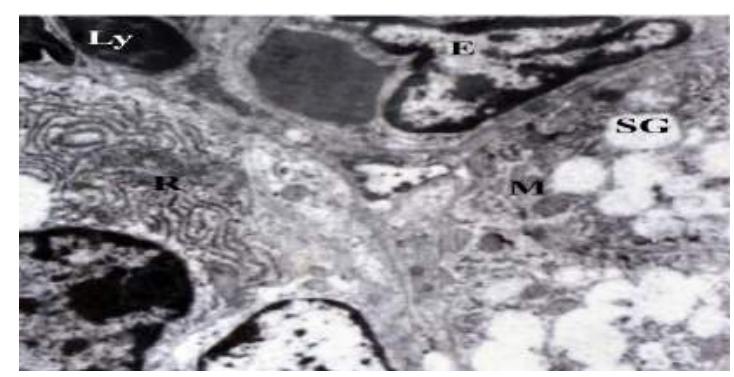

Fig.18: An electron photomicrograph of the submandibular salivary gland of rat of control group (1.1) showing endothelial cell (E) lining blood vessel and lymphocyte (Ly) in connective tissue stroma and serous acinar cell with mitochondria (M) and secretory granules (SG) . (uranyl acetale \& lead citrate×2000) 


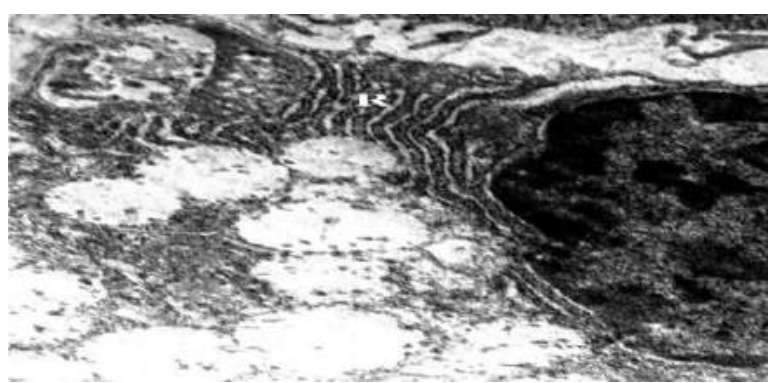

Fig. 19: An electron photomicrograph of the submandib ular salivary gland of rat of sirolimus treated group showing serous cells with marked widening of the rough endoplasmic reticulum $(R)$ and marked decrease in the electron density of their secretory

granules. (uranyl acetale\&leadcitrate×20000)

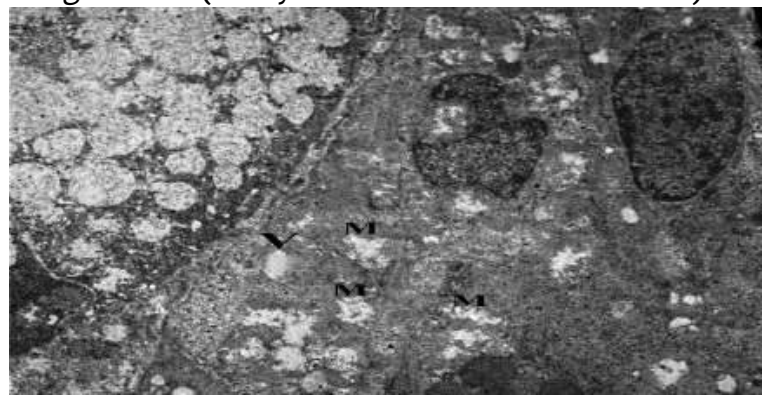

Fig. 21: An electron photomicrograph of the submandibular salivary gland of rat of sirolimus treated group showing serous cells with cytoplasmic vacuolization (V) and degenerated mitochondria (M). (uranyl acetale\&lead citrate $\times 6000)$

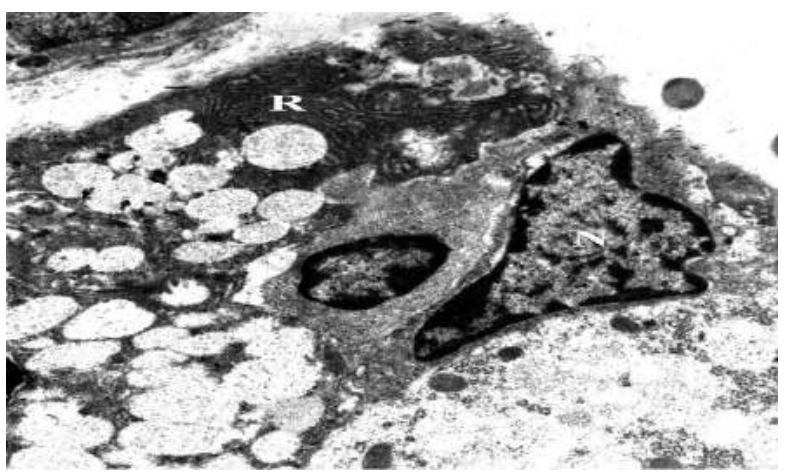

Fig.23: An electron photomicrograph of the submandibular salivary gland of rat of sirolimus treated group showing serous acinar cell with, pyknotic neucus $(N)$ dilated rough endoplasmic reticulum $(R)$ \& pleomorphism of their low electron dense secretory granules (uranyl acetale\&lead citrate×8000)

The serous acini appeared atrophic with loss of normal architecture. Sirolimus is a mTOR inhibitor that bind to an abundant

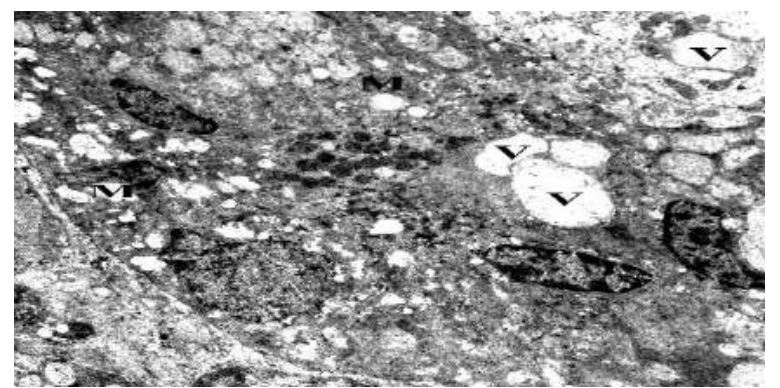

Fig. 20: An electron photomicrograph of the submandibular salivary gland of rat of sirolimus treated group showing intercalated duct with cytoplasmic vacuolization ( $V$ ) and degenerated mitochondria

(M). (uranyl acetale\&lead citrate×4000)

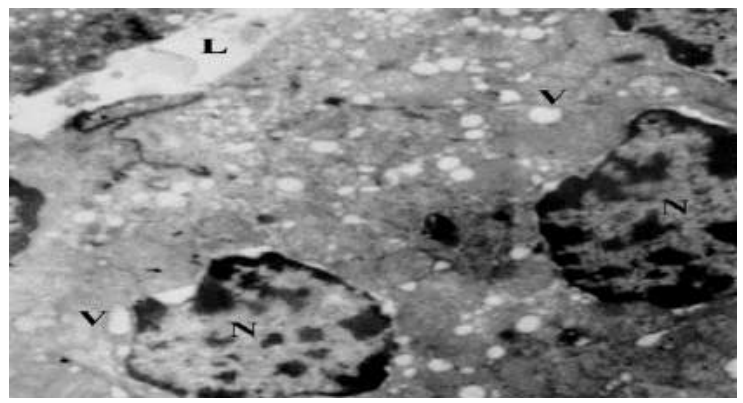

Fig. 22 An electron photomicrograph of the subman-

dibular salivary gland of rat of sirolimus treated group showing intercalated duct cells with extensive cytoplasmic vacuolization (V) irregular nuclear pattern (N) irregular lumen ( $L$ ) with stagnation of secretion.(uranyl acetale\&lead citrate×2000)

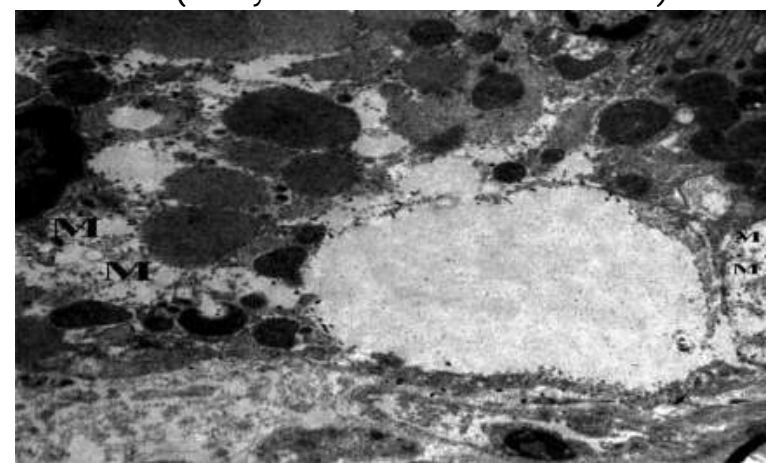

Fig. 24: An electron photomicrograph of the submandibular salivary gland of rat of sirolimus group showing degenerated mitochondria dissociation of connective tissue and widely dilated lymph vessel. (uranyl acetale \&lead citrate×8000)

intracellular binding protein FKBP-12, and mtor is an important pathway that regulate cell growth ,proliferation and 
survival(6) Suggesting that the structural and ultrastructural changes of glandular tissues may be due to direct toxicity of sirolimus by direct blocking of mtor pathway in salivary gland tissues is possible. Sirolimus has suppressive effect on immune system by inhibiting IL2 and other cytokine receptor dependent signal transduction mechanisms there by blocks activation of $T$ and $B$ lymphocytes $(7)$. The alteration of salivary gland structure and ultrastructure reported in the present investigation in sirolimus treated group may be due to viral infection of rats. sirolimus inhibition of mTORC1 appears to mediate the drug's benefits, it also inhibits $\operatorname{MTORC2}$, which results in diabetes-like symptoms (8). The later may eventually affect the structure and ultrastructure of many organs and tissues in the body, salivary glands are among them. Sirolimus has significant determinal effect on B-cell function and survival and peripheral insulin resistance this because sirolimus inhibit $m$ tor that control mRNA translation ,cellular proliferation, cell growth ,differentiation ,angiogenesis, and apoptosis and cause toxicity in B cell represented by reduction in $B$ cell size mass proliferation and insulin secretion alongside increase apoptosis (9). The connective tissue stroma of the submandibular salivary gland of the present investigation showed signs of degeneration represented by dissociation of collagen fibers, degenerated fibroblasts and markedly dilated blood vessels engorged with blood. Sirolimus also has antifibrotic effects that could have important implications for liver transplant recipients. Hepatic fibrosis is a major histologic finding in two of the most important types of recurrent disease after liver transplantation: hepatitis $C$ and primary sclerosing cholangitis., In animal models, sirolimus reduced hepatic fibrosis primarily through inhibition of hepatic stellate cell proliferation ${ }^{(10)}$. The cause of dissociation of the collagen fibers around ducts of salivary gland of sirolimus treated group may be due to the inhibitory effect of sirolimus on fibroblast growth factor and so inhibition of collagen formation. The dilatation of blood vessels of the stroma of submandibular salivary gland of rats was a constant finding in the present investigation. This arteriovenous dilatation may lead to engorgement and stagnation of blood and decrease in its flow, resulting in hypoxia and ischemia of the salivary gland tissues. Sirolimus has antiangiogenic activities linked to decrease in production of vascular endothelial growth factor and markedly inhibited response of vascular endothelial cells to stimulate $\operatorname{VEGF}^{(11)}$. Ultra structurally the mitochondria of the acinar and ductal cells appeared pleomorphic and vacuolated with total or subtotal loss of cristea and their possible rupture. These changes are expression of metabolic deterioration associated with inhibited activity of respiratory enzymes. These changes of mitochondria that appeared vacuolated, degenerated or ruptured are expression of metabolic processes associated with inhibited activity of respiratory enzymes. The execratory ducts showed atrophic epithelial lining. Others were presented with extremely thin epithelial lining, dilated lumen filled with stagnant secretion leading to accumulation of these secretions within the lumen of ducts. These changes might be due to alteration of metabolism in some ducts which may undergo degeneration due to the long period of the experiment. The dilated rough endoplasmic reticulum reported in the acinar and ductal cells of the present investigation are usually associated with reversible cell injury ${ }^{(12)}$. The cytoskeleton is a network of filaments and tubules that extends throughout a cell, through the cytoplasm. It is found in all cells.The cytoskeleton supports the cell, gives it shape, organizes and tethers the organelles, and has roles in molecule transport, cell division and cell signaling ${ }^{(15)}$. 
Alteration in any of the components of the cytoskeleton could adversely affect the normal function of the cell (13). In the present investigation monoclonal antibodies of clone LP34 was used to react with specific the cytokeratin 5,6,18. In the submandibular salivary glands of control group, the serous acinar cells give moderately positive staining reactivity to cytokeratin however the duct system revealed strongly positive staining reaction ${ }^{(14)}$. The immunohistochemical reaction of the cytokeratin 5,6,18 in the submandibular gland of the sirolimus treated group showed decreased in staining reactivity .This change in the immunohistochemical staining reactivity of cytokeratin was the result of atrophic and degenerative changes in the glandular acinar as well as the ductal cells which were noticed in electron microscope results. Any disturbance or decrease in the cytokeratin may lead to deformity in the acinar cells, defective cellular junction, leading to wide intercellular junction and defective Golgi apparatus. Any deviation from normal cytokeratin content will have an adverse effect on the glands ${ }^{(15)}$. Studies involving biopsy material from patients treated with rapamycin from the beginning proved that the drug to has a negative effect on intermediate filaments. Rapamycin caused focal lesions in podocytes, including incorrect cytokeratin expression which agrees with the present results ${ }^{(16)}$. The results of the present investigation revealed that $80 \%$ of sirolimus treated rats suffered areas of alopecia at different regions. Alopecia may be due to direct antiproliferative or antiangiogenic effect of sirolimus that leads to ischemia due to decrease blood supply which consequently results in hair loss.

\section{Conclusion}

Based on histological, immunohistochemical and ultrastructural finding we can conclude that: Sirolimus administration cause structural and ultrastructural alterations in the parenchymal and stromal elements of the submandibular salivary glands which would affect their secretory function and hence affects the health of the oral tissue and oral hygiene. These alterations be attributed to direct toxic and antiproliferative effect of the drug or due to infection causing decreased immunity. Thus, patient taking sirolimus as a medication for organ transplantation or autoimmune disease may suffer from xerostomia and decrease of salivary secretion and that may lead to determinal effect on oral health.

\section{References}

1. Khurshid Z, Haq J, Khan R.: Human Saliva and Its Role in Oral \& Systemic Health. JPDA, 2016; $25: 171$.

2. Puy $C$, Ospina A.: The role of saliva in maintaining oral health and as an aid to diagnosis. Med Oral Patol Oral Cir Bucal, 2006; 11: 449-55.

3. Folke S.: The subjective meaning of xerostomia an aggravating misery. International Journal of Qualitative Studies on Health and Well-being, 2009; 4: 245-255.

4. Geissler E Paulsson G, Fridlund B.: Immunosuppression for liver transplantation. Gut.2005 58:452-463.

5. Markus N, Schäffer M, Schier R.: Sirolimus impairs wound healing. Langenbeck's archives of surgery,2007; 392: 297-303.

6. Gresik E.: The granular convoluted tubule (GCT) cell of rodent submandibular glands. Microscopy research and technique, 1994;27:1-24.

7. Abraham R, Wiederrecht G: Immunopharmacology of rapamycin. 
Annual review of immunology, 1996;14: 483-510.

8. Sehgal S: Rapamune ${ }^{\circledR}$ (RAPA, rapamycin, sirolimus): mechanism of action immunosuppressive effect results from blockade of signal transduction and inhibition of cell cycle progression. Clinical biochemistry, 1998; 31: 335-340.

9. Barlow A, Nicholson M.: Herbert, Evidence for rapamycin toxicity in pancreatic $\beta$-cells and a review of the underlying molecular mechanisms. Diabetes, 2013;62: 26742682.

10. Debrardinis R: The biology of cancer: metabolic reprogramming fuels cell growth and proliferation. Cell metabolism, 2008; 7: 11-20.

11. Boardman $D$, Jacob $M$, Smyth $L$, Lombardi G: What Is Direct Allorecognition. Current Transplantation Reports, 2016;4: 275-283.

12. Guba M, Steinbauer M, Koehl G.: Rapamycin inhibits primary and metastatic tumor growth by antiangiogenesis: involvement of vascular endothelial growth factor. Nature medicine, 2002; 8:128.
13. D'haens G, Haens D, Bodoky G.: Early lesions of recurrent Crohn's disease caused by infusion of intestinal contents in excluded ileum. Gastroenterology, 1998. 114: 262267.

14. Caselitz J, Dardick, Parks W.: Intermediate-sized filament proteins (prekeratin, vimentin, desmin) in the normal parotid gland and parotid gland tumours. Virchows Archiv A,1981;393:273-286.

15. Schlegel R, Nash G, S Banks S: Immunohistochemical localization of keratin in normal human tissues. Laboratory investigation; a journal of technical methods and pathology, 1980; 42: 91-96.

16. Grabowska M,Słuczanowska M.: The impact of selected immunosuppressive drugs: rapamycin, cyclosporine $A$ and prednisone on immunolocalization and immunoexpression of cytokeratin in prostate gland-experimental model. journal of puplic health, 2014: 128: 7782. 